are given; not all teams follow this practice. Agreement on how frequent doses should be given and suitable maximum daily ranges would be beneficial. Some teams give more advice relating to renal failure. Few teams routinely give a Patient Information leaflet- may benefit.

\section{INPATIENTS WITH PALLIATIVE CARE NEEDS ANTICIPATORY PRESCRIBING REVIEW - UNIVERSITY HOSPITAL HAIRMYRES, NHS LANARKSHIRE}

Michaela Watt, Nicola McCann, Gillian Thomson, Karen Harvie. NHS Lanarkshire

10.1136/spcare-2020-PCC.109

Background The Scottish Palliative Care Guidelines published in 2014 outline best-practice to support clinicians with end of life care. These guidelines include recommendations on anticipatory prescribing for patients nearing the end of life to allow these people to have timely access to injectable symptom control medication.

Aim To improve anticipatory prescribing in Hairmyres Hospital in line with Scottish national guidelines through evaluation of current practice and an educational programme for prescribers.

Method Inpatient medication prescription charts were reviewed and data analysed retrospectively. Inpatients referred to the hospital palliative care team for whom anticipatory prescribing was appropriate, over a four week period $(n=20)$ were included. Data about patient demographics, anticipatory prescribing and subcutaneous infusions was collected. A teaching session on anticipatory prescribing was arranged for medical staff along with on-going case-by-case feedback and education from the palliative care team. Data was then collected in a further quality improvement cycle $(n=12)$.

Results $60 \%(n=12 / 20)$ of patients were prescribed all recommended anticipatory medications. Of these, $77 \%$ of prescriptions were in accordance with the national guidelines $(n=53$ / 68). $71 \%$ of continuous subcutaenous infusions were prescribed correctly $(n=5 / 7)$. Following the education programme, $67 \%(n=8 / 12)$ were prescribed all recommended anticipatory medications and of these, $86 \%$ of medications were prescribed in accordance with the guidelines $(n=32 / 37)$. 100\% of continuous subcutaneous infusions were prescribed correctly $(n=6 / 6)$. Conclusion This education programme improved the rate and accuracy of prescribing of anticipatory medication for patients nearing the end of life. More work is required to ensure awareness amongst prescribers of how and when to prescribe these medications with the aim of full compliance with the Scottish National Palliative Care Guidelines.

\section{RETROSPECTIVE AUDIT OF THE PRESCRIPTION OF OPIOIDS AT THE END OF LIFE IN A HOSPICE AND HOSPITAL SETTING IN THE WAKE OF THE GOSPORT INQUIRY}

Phoebe Wright, Christopher Nobbs. Meadow House Hospice

\subsection{6/spcare-2020-PCC.110}

Background The Gosport Inquiry (GI) found that unsafe opioid use led to around 450 excess deaths over 14 years. We compared their findings to local, current use in a hospice and hospital setting.
Methods Notes were reviewed retrospectively for inpatient deaths in January 2019 at Ealing Hospital and Meadow House Hospice. We collected the same data as was analysed in the GI, using notes and drug charts. We focused on indications, doses and routes of administration of opioids.

Results At the hospice, 92\% (24/26) of patients received regular opioids. $100 \%(26 / 26)$ had appropriate indications documented. The mean initial dose was $30 \mathrm{mg}$ oral morphine equivalent per day; the mean final dose was $35 \mathrm{mg}$. $62 \%$ of patients $(16 / 26)$ were switched to continuous subcutaneous opioid administration prior to death. At the hospital, 42\% (26/62) of patients received opioids. 23\% (14/62) received regular opioids, and of these 93\% (13/14) had appropriate indications documented. The mean initial dose was $20 \mathrm{mg}$ oral morphine equivalent per day; the mean final dose was $24 \mathrm{mg}$. Of total deaths, 5\% (3/62) received opioids via continuous subcutaneous infusion, 18\% (11/62) were reviewed by palliative care. The narrative in the notes suggested a lack of confidence in prescribing opioids and diagnosing dying.

Conclusions It is reassuring that opioid use at both sites appears safe. In contrast to the GI, opioid doses used were low and indications well documented. The proportion of patients receiving opioids was lower at the hospital than the hospice, with an apparent preference for low dose, immediate release rather than background opioids. The size of the difference could represent truly different patient populations, or could be under-utilisation of opioids as a result of lack of confidence or education. This signposts possibilities for future investigation as to why this might be, and potential improvements to clinical practice.

\section{ARE WE READY?}

N Wright, S Dargan, C Clackson, C Morgan, F Power, J Samarasinghe, S Henderson, C Smith. Ashford and St Peter's NHS Foundation Trust

\subsection{6/spcare-2020-PCC.111}

Introduction Annually at Ashford and St Peter's Hospital (ASPH) we hold an event to promote dying matters week. This aims to remove the stigma of talking about death and dying within our community. In 2019, dying matters week theme was 'Are we ready?'.

Methods The Specialist Palliative care team (SPCT) designed a survey to gauge if the ASPH community 'were ready?'. We asked questions including: have you cared for someone who was dying?, did you feel ready?, have you thought about future wishes? i.e. medical treatment, how would you like to be cared for?, where you would like to be cared for?, most importantly have you discussed these wishes with those important to you? The survey was distributed to all areas of the hospital.

Results 80 surveys were returned: 56 (70\%) Staff, 1 (1\%) patient, 4 (5\%) Carer/family member, 3 (4\%) Visitor, 16 (20\%) not completed. $86 \%$ were aged $20-60$ which is representative of the ASPH community. Of the respondents $57 \%$ felt ready to care for someone who was dying. However only $45 \%$ had thought about how they would like to be medically treated at the end of life (EOL) and 38\% knew where they would like to be cared for at the EOL. Only $50 \%$ of respondents had discussed this with those important to them. 
Conclusions As a healthcare community we are faced with the challenges of illness, uncertainty and death every day. However we are poor at considering our future EOL care. Despite having an awareness of the importance of sharing our thoughts with our loved ones we only do this half the time. By openly talking about death and dying we encourage our community to do the same. The hope is this will allow us to do better when talking to our patients.

\section{Ethics | posters $92-95$}

\section{ETHICAL CHALLENGES OF ARTIFICIAL INTELLIGENCE TECHNOLOGY IN PALLIATIVE CARE}

Matthew Cavaciuti, Amara C Nwosu. University of Liverpool, Palliative Care Institute Liverpool, Liverpool University Hospitals NHS Foundation Trust, Marie Curie Hospice Liverpool

\subsection{6/spcare-2020-PCC.112}

Background Artificial Intelligence (AI) is an area of computer science which involves the development of intelligent machines that work and react like humans. AI has potential to improve healthcare delivery through purposeful analysis of clinical record data. Examples of AI use in palliative care includes the analysis of electronic patient record data to predict survival, classify pain severity and to identify important clinical discussions. Despite the opportunities of AI, there are a number of ethical challenges of using this technology in palliative care. Consequently, this study aimed to identify the ethical challenges of AI in palliative care.

Methods A narrative scoping review of literature was undertaken to identify the evidence of AI use in palliative care. Three real-world case studies using AI in palliative care were critiqued in depth, using the four ethical principle framework (Autonomy, Justice, Beneficence, Non-maleficence). Ethical challenges were identified and summarised into themes.

Results Very few studies have examined the use of AI in palliative care; no studies discuss the ethical challenges as the primary focus. Ethical challenges for AI in palliative care were summarised into four themes: (1) Data privacy and security; (2) Artificial stupidity; (3) Prognostication; (4) Unexpected results and bias.

Conclusions AI has potential to support delivery in palliative care; however, a number of important ethical challenges need to be considered. AI healthcare data analysis should be built around an ethical framework. This is important in palliative care as individuals may be more vulnerable compared to other specialities. Research to determine the views and opinions of a patients, caregivers and healthcare professionals is urgently needed. Our work has led to the development of recommendations for ethical AI research in palliative care, which will hopefully guide meaningful use of this technology.

\section{IN YOUR OPINION WHAT ARE THE MOST IMPORTANT ETHICAL ISSUES FOR THE SPECIALTY IN THE NEXT FIVE YEARS AND HOW COULD WE ADDRESS THEM}

Jack Maddicks. University College London (UCL)

10.1136/spcare-2020-PCC.113
Introduction Just access to palliative care for people from Black, Asian and Minority Ethnic (BAME) populations has long been recognised as a prominent ethical issue in the specialty. However, changing social, political and demographic factors mean that this issue is especially significant now.

Methods This essay reviews literature and population data to explore the barriers faced by people from these groups in the UK. They are grouped into patient-level, society-level and structural barriers, with provider-level mediators.

Findings There are numerous patient-level, personal barriers in palliative care. Frequently denoted examples include cultural, religious and linguistic differences between patient and caregiver. Although the exact nature of these barriers varies between ethnic groups, their existence remains relatively unchanged over time. However, recent society-level factors - the demographic changes of an ageing, diversifying, mobile population - is magnifying their impact currently. Structural changes also lend the issue contemporary significance. Namely, the so-called 'hostile environment' policies of recent governments have produced new barriers to palliative care. Although palliative care is exempt from patient charging, and the policies do not specifically target BAME populations, both are still affected. Poor implementation has led to inappropriate identity challenges, hospital fees and denial of care, which has in turn worsened healthseeking behaviour and healthcare experience amongst people from BAME groups.

Suggested solutions The solutions suggested are rooted in medical education: the provider-level factors that mediate barriers to care. These include 'structural competencies' to encourage engagement of clinicians in political discourse, and 'cultural competencies' to facilitate greater understanding between clinicians and patients. Inclusion of these extra-clinical competencies into training would create a healthcare workforce that is able to commission equitably, engage with policy and care effectively.

\section{ENABLING DYING PATIENTS TO TAKE PART IN RESEARCH USING ADVANCED CONSENT METHODOLOGY}

Sarah Stanley, Amara C Nwosu, Laura Chapman. Marie Curie Hospice Liverpool, Palliative Care Institute Liverpool, University of Liverpool

\subsection{6/spcare-2020-PCC.114}

Background Palliative care research is essential to establish evidence based models of care that are required to improve outcomes for people with terminal illness. Obtaining informed consent for participation in hospice and palliative care clinical trials is an ongoing challenge faced by researchers.

Aim By consenting patients in advance, we aimed to improve research opportunities in the last week of life.

Methods Since 2017, an advanced consent methodology was used to recruit participants to two end of life care studies (UK National Institute for Health Research Portfolio study) from a specialist palliative care inpatient unit. Potential participants with capacity were given information by a researcher, which outlined the study aims and the advanced consent process. This included explanation of the end of life assessments. A 'Personal consultee' was 